\title{
Classical Conditioning of the Eye Withdrawal Reflex in the Green Crab
}

\author{
Charles I. Abramson and Richard D. Feinman \\ Department of Biochemistry, State University of New York Health Science Center at Brooklyn, Brooklyn, New York 11203
}

Eye withdrawal in the green crab, Carcinus maenas was conditioned by pairing a mild vibration to the carapace as a conditioned stimulus (CS) with a puff of air to one of the eyes as an unconditioned stimulus (US). Animals subjected to repeated pairings showed an increased probability of eye retraction during $C S$ presentation. Significantly less responding was found in several control groups subjected to backward conditioning, unpaired stimuli, stimuli alone, or simply time in the apparatus. Although conditioned animals showed few responses to CS alone after $24 \mathrm{hr}$, retention could be demonstrated by acquisition that was much more rapid on day 2 than on day 1 . Conditioning could also be effected in the eye when it was restrained, a result consistent with reports in the literature that this reflex does not require proprioceptive feedback. Because the neuromuscular circuitry of eye withdrawal is already well defined in Carcinus, this is a promising candidate for studying the neuronal basis of classical conditioning.

The eye withdrawal response of the crab is a classic example of a defensive reflex. The system is well characterized anatomically and physiologically and a number of simplifying features make it attractive for the study of neuronal correlates of learning. We have previously shown that extension of the eye can be repressed by a simple kind of operant procedure using contingent punishment (Abramson and Feinman, 1987), and in this report we describe a classical (Pavlovian) conditioning procedure in which animals are taught to associate an initially neutral stimulus, mild vibration on the carapace, with an aversive puff of air to the eye. As a potential model for the study of the neuronal substrates of learning, this system has the advantage that much of the neuromuscular circuitry has already been described (Burrows, 1967; Sandeman, 1967; Burrows and Horridge, 1968a, b). Only 2 motor neurons mediate the retraction, and the withdrawal reflex overrides other more complex behavior of the eyes such as the optokinetic response. The physiology is further simplified by the fact that there is no proprioceptive feedback.

In the training used here, repeated pairings of vibration as a conditioned stimulus (CS) with an unconditioned stimulus (US), a puff of air to one of the eyes results in the reliable appearance of the response, retraction of the eye into the carapace during CS presentation. We found significantly less responding in con-

\footnotetext{
Received Sept. 7, 1987; revised Dec. 9, 1987; accepted Dec. 29, 1987.

We are grateful to Ms. Jacqueline Ryzman for expert technical assistance. This work was supported, in part, by funds from James C. Marlas and from the Research Foundation of the State University of New York.

Correspondence should be addressed to Dr. Charles I. Abramson, Box 8, Department of Biochemistry, SUNY Health Science Center, 450 Clarkson Avenue, Brooklyn, NY 11203.

Copyright (C) 1988 Society for Neuroscience $0270-6474 / 88 / 082907-06 \$ 02.00 / 0$
}

trol groups subjected to backward conditioning or specifically unpaired presentation of stimuli. There were also no anticipatory responses to US alone presentations, and we observed little effect on control animals of presentation of the CS alone or simply time in the apparatus. Retention of the association was maintained for at least $24 \mathrm{hr}$ as determined by reacquisition.

A preliminary description of some of this work was presented in abstract form (Feinman and Abramson, 1987).

\section{Materials and Methods}

Male crabs of mean carapace width of $4.5 \mathrm{~cm}$ (housed for 2 weeks prior to use) were selected at random, wrapped in a paper towel soaked in seawater, and restrained in a standard laboratory clamp as described previously (Abramson and Fcinman, 1987). The CS was a $5 \mathrm{scc}$, lowamplitude vibration conducted through a $3 \mathrm{~cm}$ stove bolt attached to a buzzer. The tip of the bolt rested on the carapace $4 \mathrm{~mm}$ behind the eye that was to be conditioned. For half the animals the left eye was trained. For the remaining subjects, the right eye was trained. The US was a $0.5 \mathrm{sec}$ puff of air $(0.25 \mathrm{psi})$ directed at the eye through a nozzle. The US invariably caused retraction of the eye into the carapace. We used an experimental group of 8 animals (paired presentations of CS and US) and 5 control groups of 8 animals each (unpaired, backward, CS only, US only, and blank, as described below). Subjects were assigned to experimental or control conditions at random. Animals received 100 trials and, to avoid possible fatigue, every 20 th trial was followed by a $10 \mathrm{~min}$ rest period during which they were placed, unrestrained, in seawater. The experimental group received 100 pairings of the CS and US. The CS was presented for $5 \mathrm{scc}$, at the end of which the US was presented for $0.5 \mathrm{sec}$. The intertrial interval for all animals was an average of $1 \mathrm{~min}$ (presented in intervals of random duration between $0.2 \mathrm{sec}$ and $2 \mathrm{~min}$ to prevent any fortuitous temporal conditioning). The unpaired group received $100 \mathrm{CS}$ and US presentations in a random sequence so that there were no more than 2 consecutive stimuli of the same type. Backward controls received stimuli on the same schedule as experimentals but in reverse order (US precedes CS). Blank animals were simply placed in the apparatus for the equivalent of 100 trials and and did not receive any stimuli. Two other groups received CS alone or US alone presentations. Responses were recorded manually. Approximately $50 \%$ of the data were scored blind. No differences in judgments were found between blind and informed observers. Conditioned responses were scored for full retractions of the eye resembling the unconditioncd rcsponse; small twitches were not scored. For experimentals, unpaired, CS alone, and backward groups, responses during CS presentations were recorded. For blank and US alone animals, responses in the $5 \mathrm{sec}$ interval during which the CS would normally be presented were recorded. After training trials, all groups received 20 extinction trials in which the CS was presented alone. Ten minutes after the extinction trials, the paired, unpaired, and backward groups received $20 \mathrm{CS}$ alone trials presented to the eye that had not been used in training. This eye was rarely retracted during the conditioning period.

\section{Results}

Demonstration of classical conditioning of the eye withdrawal reflex

The effects of vibration/air puff (CS-US) pairings and of the 5 control procedures on groups of 8 crabs are shown in Figure 1. 


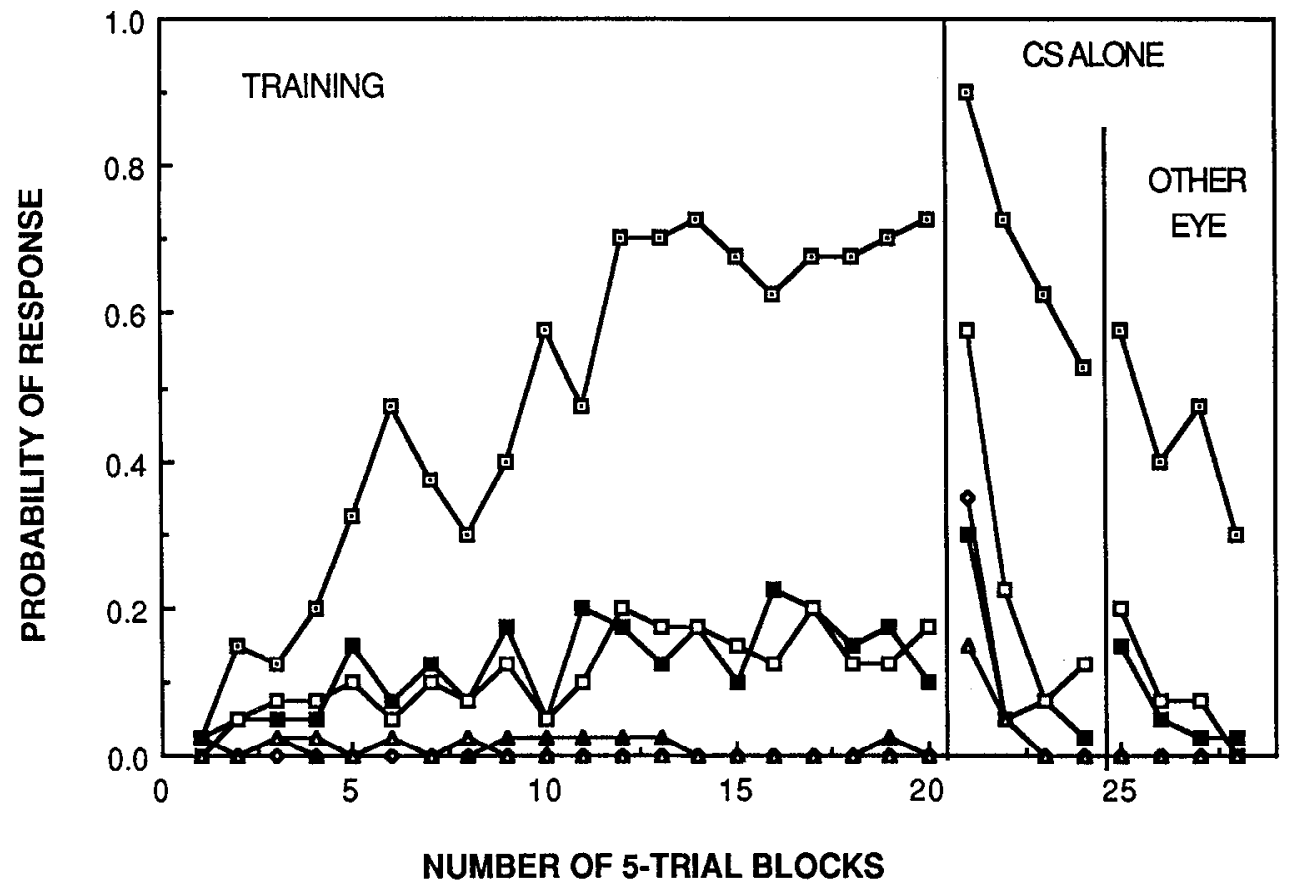

Figure 1. Probability of response during CS presentation. Groups of 8 experimental (paired) animals or controls were subjected to training as indicated, and the responses of all animals in each group are averaged for blocks of 5 trials. The experimental groups were as follows: paired (open squares with dots), unpaired (filled squares), backward (open squares), US alone (open diamonds), CS alone (filled triangles), blank (open triangles). Immediately after training, the CS alone was presented first to the trained eye and then to the contralateral eye.

It is evident that, in the paired group, the mean probability of a response to the CS increased with training. Both the backward and unpaired groups showed an increase in responding during the session, but this was not as pronounced as the paired group. The increase in response to the CS in these control groups was presumably due to the sensitizing effect of multiple presentation of the stimuli, but we cannot exclude the possibility that, in the first case, backward conditioning was actually taking place, and, in the second, that the "unpaired" animals actually received fortuitous pairings (since we do not know the precise dependence of conditioning on interstimulus interval). Figure 1 also shows that there was no effect on the behavior of the control groups subjected to repeated exposure to CS, US, or time in the experimental situation. Figure 2, which summarizes the total number of responses for all groups, demonstrates that the population behavior is a true reflection of individual performance. Although there were variations, each individual animal subjected to paired training showed significant increases in responding. Only one animal of the control groups made more responses than the lowest individual in the paired group. These conclusions are further borne out by a statistical analysis. A KruskalWallis nonparametric analysis of variance based on the number of conditioned responses by each subject in each block of 5 trials and modified to test for main effects and interactions (Bradley, 1968) showed that, as the session progressed, CS responses significantly increased in the paired, unpaired, and backward groups $(H=26.142, d f=7, p<0.001)$. The number of $C S$ responses differs among the 3 groups $(H=14.473, d f=2, p<0.001)$, and there was a significant group $\times$ trial interaction $(H=47.46$, $d f=14, p<0.001)$. Post hoc comparisons conducted with the Mann-Whitney $U$ test revealed no difference between backward and unpaired groups $(U-29, p-0.399)$ but highly significant differences between paired and unpaired groups $(U=2, p=$
$0.000)$ and between paired and backward groups $(U=0, p=$ 0.000 ). That there was no significant increase in the CS, US, or blank groups was confirmed by a Kruskal-Wallis ANOVA, which revealed no significant group effect $(H=8.611, d f=2, p>$ $0.98)$, trial effect $(H=10.567, d f=7, p>0.20)$, or group $\times$ trial interaction $(H=18.806, d f=14, p>0.20)$.

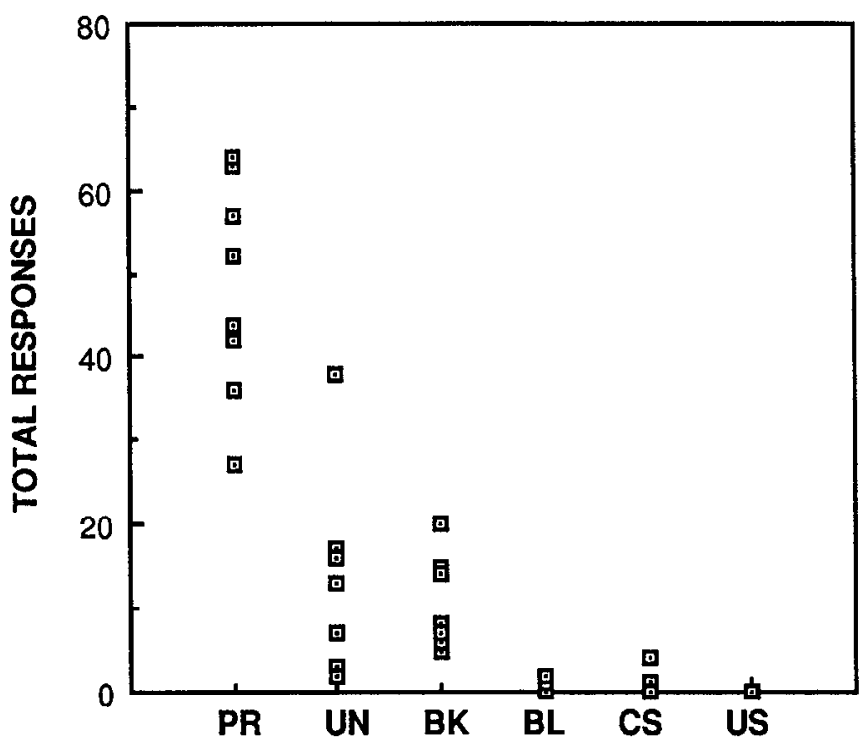

Figure 2. Total responses of individual animals during the training period shown in the first panel of Figure 1. A maximal score of 80 is possible. Each group has 8 animals, and some scores overlap. Subjects were presented with paired stimuli $(P R)$, unpaired $(U N)$, backward pairing $(B K)$, no stimuli $(B L)$, or CS or US alone. 
The difference in effects between the paired and control procedures can also be seen in the performance of these groups during extinction. The paired and backward control groups, when tested for responses to CS alone, extinguished fairly rapidly. The paired group, in distinction, responded at a high level and did not reach the low levels obtained by the control. There was also high variability in the extinction performance of the paired animals: 5 out of 8 still responded at a high level through the 20th CS only presentation. This variability was confirmed by a Kruskall-Wallis ANOVA, which showed no trial effect ( $H$ $=10.611, d f=7, p>0.05$ ). However, there were significant differences among the 3 groups in terms of the number of extinction response $(H=13.659, d f=2, p<0.01)$. There was also a significant group $\times$ trial interaction $(H=29.14, d f=14$, $p<0.001)$. Post hoc comparisons revealed significant differences between paired and unpaired groups $(U=2.5, p=0.001)$, paired and backward groups $(U=6.5, p=0.003)$, and unpaired and backward groups $(U=13, p=0.025)$. The latter finding is of particular interest because the performance of unpaired and backward groups was not statistically different during acquisition $(p(U)=0.399)$ but was during extinction. A plausible explanation for this difference in extinction but not in acquisition is that, for backward animals, the presentation of the CS without the prior occurrence of the US is a novel event that might produce sensitization (see, e.g., Abramson and Bitterman, 1986). This is consistent with the performance of the blank and US alone control groups, which made several responses to the CS during the first 5 trials even though they never received the CS during the previous 100 trials of the experiment (Fig. 1).

At the end of the training and extinction periods, the contralateral eye of each animal of the paired, unpaired and backward groups was tested for $\mathrm{CS}$ responses. Figure 1 shows that paired animals made several responses, although the CS had never been paired with air puff in this eye. This behavior is reminiscent of the transfer of learning to the contralateral eye observed in the nictitating membrane response of the rabbit, a learning paradigm with which the current study has a philosophical kinship (Rosenfield and Moore, 1985). The data in the figure are averages for the animals trained, although 1 of the 8 made no responses during CS presentations to the untrained eye. We also found that untrained eyes of 3 subjects of the unpaired group and 4 subjects of the backward group responded to CS alone presentations. Kruskal-Wallis ANOVA revealed significant differences between the number of CS responses of the contralateral eye of the paired, unpaired, and backward groups $(H=10.865$, $d f=2, p<0.01$ ), and, as the number of CS presentations increased, there was a significant decline in the number of responses $(H=18.093, d f=7, p<0.02)$. There was also a significant group $\times$ trial interaction $(H=18.582, d f=7, p<$ 0.01 ). Post hoc comparisons revealed no differences between unpaired and backward groups $(U=28, p=0.360)$ and highly significant differences between paired and unpaired groups $(U$ $=5, p=0.001)$ and paired and backward groups $(U=7, p=$ 0.003 ). A correlational analysis revealed that the probability of responses of the eye not used in training was related to the number of responses elicited during extinction (Kendall $R=$ $0.61, Z=4.176, p<0.001)$.

\section{Retention of the conditioned response}

We carried out a second series of experiments to assess the retention of the classically conditioned eye withdrawal. The material, methods; apparatus, random selection of subjects, and housing conditions were identical to those employed in Experiment 1. Two measures of retention were investigated: (1) CS alone and (2) reconditioning. In the CS alone measure, 8 new animals were selected at random and given $100 \mathrm{CS}$-US pairings. After training they were placed individually in plastic containers for $24 \mathrm{hr}$, at which time they were returned to the conditioning apparatus and given $50 \mathrm{CS}$ only presentation with an average intertrial interval of $1 \mathrm{~min}$. Figure $3 A$ (open squares) shows the performance of this group in the training session on day 1 . Figure $3 B$ shows that when these animals were tested on day 2 for CS only responses, there was little evidence of retention of the conditioned response. We therefore turned to reacquisition as a test of memory.

In the retention experiments, a new group of 8 animals was given 100 training trials, isolated for $24 \mathrm{hr}$ and then given 100 more training trials. The performance of this group was compared with their performance on day 1. The results, in Figure $3 A$ (filled squares), show a substantially enhanced rate of acquisition during the second session. Friedman ANOVA modified to test for main effects and interactions (Bradley, 1968) showed a significant trial effect between session 1 and session $2\left(X_{r}^{2}=\right.$ 50.363, $d f=7, p<0.001$ ), a significant increase in CS responses in session 2 compared with session 1 (Wilcoxon $Z=-2.521$, $p=0.0059)$, and a highly significant group $\times$ trial interaction $\left(X_{r}^{2}=41.163, d f=14, p<0.001\right)$.

In order to show that the enhanced performance in the second session on day 2 for the experimental animals was pairingspecific, we included 2 control groups subjected to paired training after unpaired or backward procedures on day 1 . The first control group received 100 unpaired CS, US presentations during a training session on day 1 followed, $24 \mathrm{hr}$ later, by 100 paired trials. A second group received 100 hackward conditioning trials followed, $24 \mathrm{hr}$ later, by 100 paired trials. All control subjects were housed individually during the $24 \mathrm{hr}$ intersession interval. Figure $3 A$ shows the second day performance of these control animals trained (on day 1) to unpaired stimuli (squares with crosses) or to backward conditioning (squares with dots). There is no significant difference between the day 2 acquisition for these control animals and the day 1 performance of the experimental group. This conclusion is borne out by a statistical analysis, which also includes the day 1 behavior of the first group of animals described above (open squares) as an additional control. Kruskal-Wallis ANOVA revealed no overall group differences between the session 1 performance of experimentals and the session 2 performance of the 3 control groups $(H=2.411, d f=2, p>0.30)$. A significant trial cffcct $(H=$ $15.109, d f=7, p<0.05)$ and a significant group $\times$ trial interaction was observed $(H=119.754, d f=14, p<0.001)$.

It is also important to mention that the first session performance of these 2 control groups (omitted from Fig. 3 for clarity) were similar to animals trained under identical conditions in Experiment 1 (see Figs. 1 and 2). This is confirmed by a KruskalWallis ANOVA, which indicated a significant group effect $(H$ $=13.424, d f=2, p<0.01$, trial effect $(H=19.879, d f=7, p$ $<0.01)$, and group $\times$ trial interaction $(H=36.393, d f=14, p$ $<0.001)$. Post hoc analysis showed significant differences between paired and unpaired animals $(U=2, p=0.000)$ and paired and backward animals $(U=2.5, p=0.001)$ but not between backward and unpaired animals $(U=27.5, p=0.323)$. This pattern of results is identical to that obtained in the acquisition phase of Experiment 1. This, in effect, constitutes a 


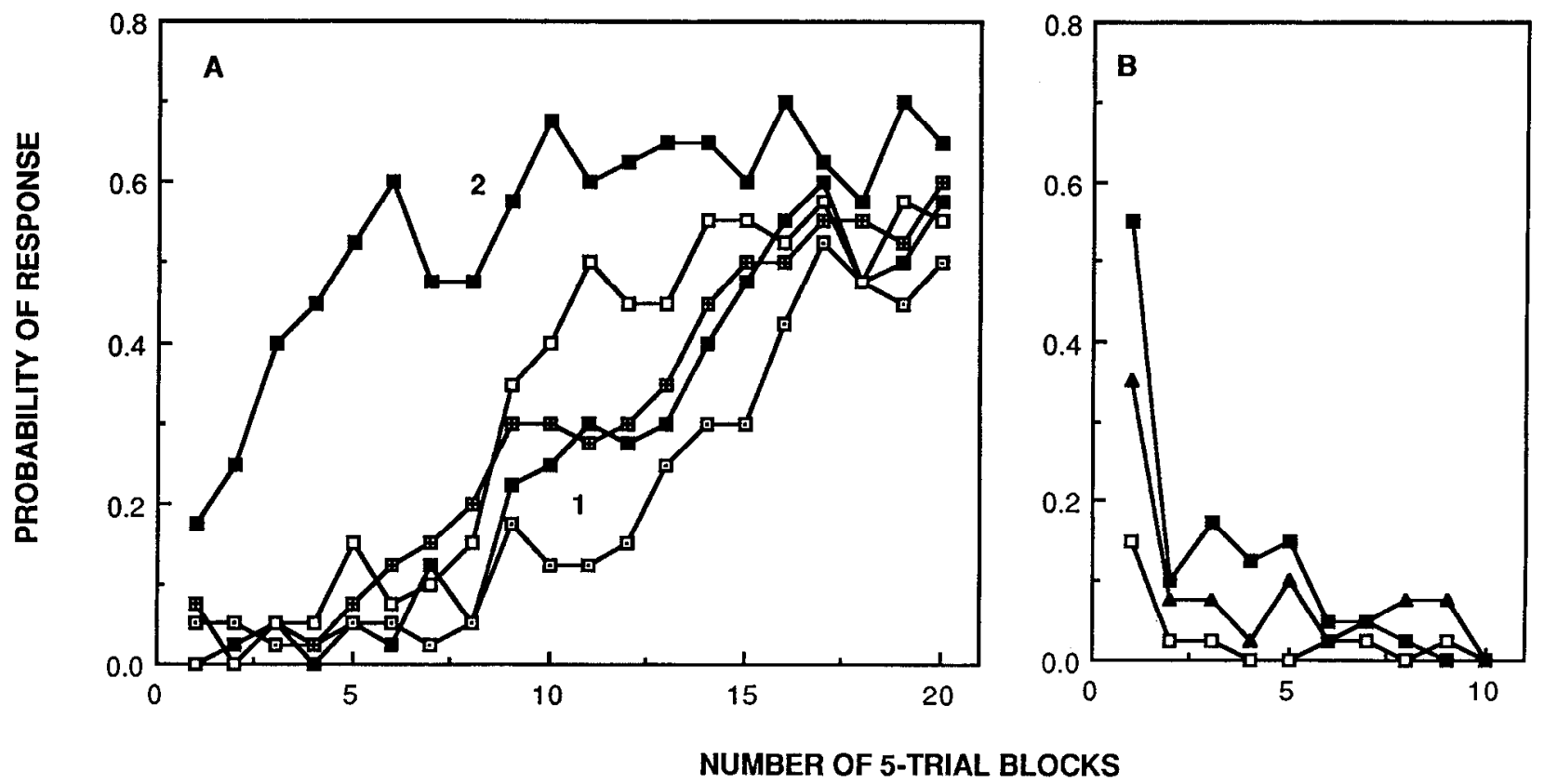

Figure 3. Retention of the effect of paired training after $24 \mathrm{hr}$. A, Retention measured by reacquisition. A group of 8 animals was trained by paired stimuli on day 1 (filled squares, 1) and then retrained on day 2 (filled squares, 2). The performance on day 2 of control animals subjected to unpaired stimuli on day 1 (squares with crosses) or backward conditioning of day 1 (squares with dots) is also shown. The performance of an additional group of 8 animals subjected to paired training on day 1 is shown as open squares. $B$, Retention measured by response to CS alone. The group of animals in $A$ with $1 \mathrm{~d}$ of training (open squares) was tested for responses to CS alone presentation after $24 \mathrm{hr}$. The group in $A$ subjected to $2 \mathrm{~d}$ of training (filled squares) was tested on the third day. A final group that had $2 \mathrm{~d}$ of time in the apparatus, but no stimuli, was also tested (filled triangles).

second demonstration of the primary effect of the classically conditioned eye response.

In summary, Figure $3 A$ shows that $24 \mathrm{hr}$ after paired training, acqusition is more rapid than on the first day, but that $24 \mathrm{hr}$ after backward or unpaired training, there is no enhancement of acquisition.

To assess the effect of continued training, we further tested the retention in the animals that had received $2 \mathrm{~d}$ of paired training by testing them on the third day with CS only. The same animals used in the reacquisition test were again housed in plastic containers for $24 \mathrm{hr}$ and then given $50 \mathrm{CS}$ only trials. This group was contrasted with another that served as a sensitization control. Here, subjects were placed in the conditioning apparatus with no stimuli for the duration of 100 training trials for 2 sessions prior to receiving $50 \mathrm{CS}$ alone trials. Like animals receiving $2 \mathrm{~d}$ of paired training, the sessions were separated by $24 \mathrm{hr}$. Figure $3 B$ shows the results of the CS alone retention experiment in terms of the probability of response over successive 5-trial blocks. There was significantly more responding in the group with $2 \mathrm{~d}$ of pairing compared with the original group with $1 \mathrm{~d}$ of training (open squares), although most of the conditioned responses were confined to the first 5 trials, suggesting that success of our CS alone measure of retention is a function of the number of training trials. On the other hand, animals receiving 2 sessions of blank training also responded substantially to the CS when first introduced, indicating that sensitization rather than memory per se can account for the observed CS alone responses. Whether the response in the paired group contains a component of this sensitization or whether these are 2 different effects is not known. The Mann-Whitney $U$ test confirmed that performance after $2 \mathrm{~d}$ of training is significantly different from that after $1 \mathrm{~d}(U=10, p=0.010)$ but not different from behavior of the group with $2 \mathrm{~d}$ of blank ( $U$ $=23, p=0.191$ ). The fact that the differences are due to early trials is shown by an overall Kruskal-Wallis ANOVA, which revealed no significant group effect for the 3 groups $(H=5.733$, $d f=2, p>0.05)$, a significant effect of trials $(H=16.422, d f$ $=7, p<0.05)$, and a signiticant group $\times$ trial interaction $(H$ $=39.691, d f=14, p<0.001)$.

\section{Conditioning of the restrained eye}

The eye withdrawal reflex is known to proceed without proprioceptive feedback (Horridge and Sandeman, 1964; Burrows and Horridge, 1968a, b), suggesting that movement of the eye should not be required for conditioning. We tested this prediction in 6 animals by restraining the eye in the retracted position with a rubber band and subjecting it to the usual 100 pairings of CS and US as described above. Conditioning was then tested by freeing the eye and measuring the response to 20 presentations of CS alone as in the second panel of Figure $1 \mathrm{~A}$. The results are shown in Figure 4, where the behavior of animals with restrained eyes (data points) is compared with that of animals with free eyes (line) from Figure $1 A$. It is evident that conditioning can be effected in a restrained eye. The control group $(n=6)$, again with restrained eyes, but presented with unpaired stimuli gave few responses (after the cyc is frecd) in this CS-only test; the data points are, again, shown fit to the line from the corresponding animals with freely moving eyes. The fit of the data in Figure 4 is fortuitously good; the restrained animals, like their free-eye counterparts, showed great variation in the later trials (10-20). Two of the animals from the restrained population were surprisingly better in the CS test animals conditioned with freely Inoving eyes. These 2 subjects showed $90 \%$ probability of responding throughout the 20 trials, and when tested for 80 ad- 


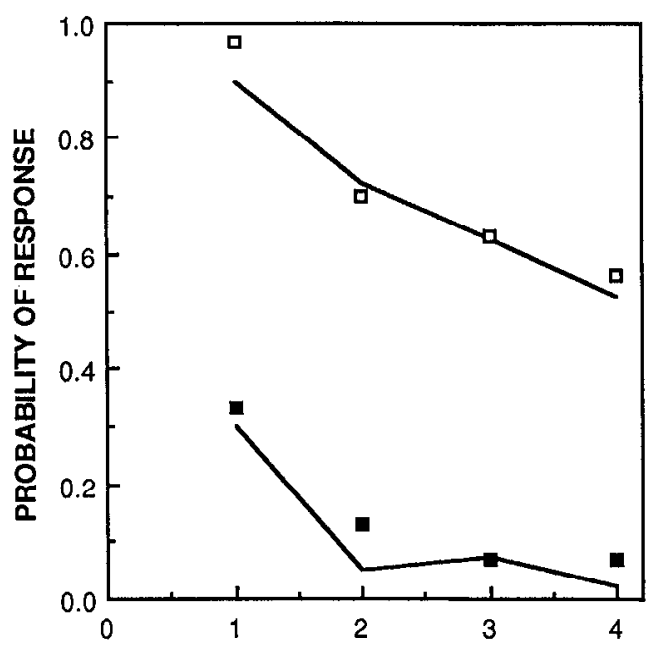

NUMBER OF 5-TRIAL BLOCKS

Figure 4. Probability of response to CS alone presentation of animals trained with eye immobilized. The eye of the animal was restrained, and the training procedure was carried out according to the paired paradigm (open squares) or the unpaired paradigm (flled squares). The eye was then freed, and responses were measured as in the second panel of Figure 1. Data are averages of 6 animals. The lines are from the second panel of Figure 1 for the corresponding procedure in unrestrained animals.

ditional trials performed at about $80 \%$ probability of response. Kruskal-Wallis ANOVA on the groups with restrained eyes revealed highly significant differences between paired and unpaired $(U=0, p=0.001)$ and no significant trial effect $(H=$ 10.836, $d f=3, p>0.05)$ and no interaction $(H=1.085, d f=$ $3, p>0.05)$. Preliminary evidence from electromyographic records of conditioning (Feinman et al., 1987) also shows that the time course of conditioning in the immobilized eye is the same as that in the freely moving eye. Thus, prima facie evidence shows that, as predicted by the physiology of this response, movement of the eye is not required for learning. It is important to point out, however, that other neurons than those studied by earlier workers may be involved in conditioning, and one cannot exclude the influence of nonmovement afferents activated by the muscle contractions.

\section{Discussion}

Lcarning phenomena that are formally identical to classical (Pavlovian) conditioning have been demonstrated in several invertebrate species (e.g., Mpitsos and Davis, 1973; Crow and Alkon, 1978; Carew et al., 1981; Sahley et al., 1981; Bitterman et al., 1983; Tully and Quinn, 1985; Abramson, 1986; Lederhendler et al., 1986; for reviews, see Farley and Alkon, 1985; Carew and Sahley, 1986; Menzel and Bicker, 1987). There are several virtues to the preparation described here. First, the conditioned behavior is easy to observe and quantify, and it permits a trial-by-trial analysis of acquisition and extinction. In addition to the accessibility of the behavior, this preparation takes advantage of a body of knowledge that already exists on the physiology of eye withdrawal. Several features of this physiology make this system a promising candidate for the study of the underlying neuronal substrate of the learned response. Analysis of the eye withdrawal is simplified by the fact that it overrides more complex behaviors such as the optokinetic response (Burrows and Horridge, 1968b). In addition, our ability to condition the restrained eye shows that the open-loop nature of the reflex (Burrows and Horridge, 1968a) extends to the learning situation. It is also of interest that the main retractor muscle, $19 a$, is innervated by only 2 motor neurons: the fast main retractor neuron that runs in the optic tract and a neuron from the oculomotor nerve, which shows a characteristic slow tonic firing pattern when the eye is retracted but is otherwise silent (Horridge and Sandeman, 1964; Burrows, 1967; Burrows and Horridge, $1968 \mathrm{a}, \mathrm{b})$. Preliminary studies of myographic activity in muscle 19 a show that the conditioning can be observed as development of the appearance of characteristic pattern due to the fast neuron even in a preparation in which the eye is not moving (Feinman et al., 1987), further supporting our observation that proprioceptive feedback is not required for conditioning. It was also observed that there are changes in the activity of the slow retractor neuron. This is significant in that activity in this neuron would be expected to reflect or be the site of the previously demonstrated operant punishment procedure (Abramson and Feinman, 1987). Thus, this preparation may allow comparison of the neuronal features of classical and operant training in the same motor system.

In summary, we have demonstrated classical conditioning of the eye withdrawal reflex of the green crab. Acquisition is reliable and relatively rapid, and there is retention for $24 \mathrm{hr}$, at least as measured by relearning. To our knowledge, with the exception of isolated reports in the early literature (review, Krasne, 1972), this is the first demonstration of a Pavlovian procedure using Crustacea. Thus, the results expand the range of invertebrate animals in which classical conditioning can be studied and provide a new simple system in which to investigate learning.

\section{References}

Abramson, C. I. (1986) Aversive conditioning in honeybees (Apis mellifera). J. Comp. Psychol. 100: 108-116.

Abramson, C. I., and M. E. Bitterman (1986) The US-preexposure effect in honeybees. Anim. Learning Behav. 14: 374-379.

Abramson, C. I., and R. D. Feinman (1987) Operant punishment in the green crab, Carcinus maenas. Behav. Neural Biol. 48: 259-277.

Bitterman, M. E., R. Menzel, A. Fietz, and S. Schaefer (1983) Classical conditioning of proboscis extension in honeybees (Apis mellifera). J. Comp. Psychol. 97: 107-119.

Bradley, J. V. (1968) Distribution-Free Statistical Tests, Prentice-Hall, Englewood Cliffs, NJ.

Burrows, M. (1967) Reflex withdrawal of the eyecup in the crab Carcinus. Nature 215: 56-57.

Burrows, M., and G. A. Horridge (1968a) Motoneurone discharges to the eyecup muscles of the crab Carcinus. J. Exp. Biol. 49: 251-267.

Burrows, M., and G. A. Horridge (1968b) Eyecup withdrawal in the crab Carcinus, and its interaction with the optokinetic response. J. Exp. Biol. 49: 285-297.

Carew, T. J., and C. Sahley (1986) Invertebrate leaming and memory: From behavior to molecules. Annu. Rev. Neurosci. 9: 435-486.

Carew, T. J., E. T. Walters, and E. R. Kandel (1981) Classical conditioning in a simple withdrawal reflex in Aplysia california. J. Neurosci. 1: 1426-1437.

Crow, T. J., and D. L. Alkon (1978) Retention of an associative behavioral change in Hermissenda. Science 201: 1239-1241.

Farley, J., and D. L. Alkon (1985) Cellular mechanisms of learning, memory, and information storage. Annu. Rev. Psychol. 36: 419-494.

Feinman, R. D., and C. I. Abramson (1987) Demonstration of classical conditioning of eye withdrawal in the green crab. Soc. Neurosci. Abstr. 13: 814 .

Feinman, R. D., C. I. Abramson, and R. R. Forman (1987) Electromyographic recording of classical conditioning in the green crab. Biol. Bull. 172: 437.

Horridge, G. A., and D. C. Sandeman (1964) Nervous control of the 
optokinetic responses in the crab Carcinus. Proc. R. Soc. London [Biol.] 161: 216-246.

Krasne, B. F. (1972) Learning in Crustacea. In Invertebrate Learning, Vol. 2, W. Corning, J. Dyal, and A. O. D. Willows, eds., pp. 49-130, Plenum, New York.

Lederhendler, I., S. Gart, and D. L. Alkon (1986) Classical conditioning of Hermissenda: Origin of a new response. J. Neurosci. 6: 1325-1331.

Menzel, R., and G. Bicker (1987) Plasticity in neuronal circuits and assemblies of invertebrates. In The Neural and Molecular Bases of Learning, J.-P. Changeux and M. Konishi, eds., pp. 433-472, Wiley, New York.

Mpitsos, G. J., and W. J. Davis (1973) Learning: Classical and avoidance conditioning in the mollusk Pleurobranchaea. Science 180:317320.
Rosenfield, M. E., and J. W. Moore (1985) Red nucleus lesions impair acquisition of the classically conditioned nictitating membrane response but not eye-to-eye savings or unconditioned response amplitude. Behav. Brain Res. 17: 77-81.

Sahley, C., J. Rudy, and A. Gelperin (1981) An analysis of associative learning in a terrestrial mollusc. I. Higher-order conditioning, blocking, and a transicnt US pre-cxposurc cffcct. J. Comp. Physiol. 144. $1-8$.

Sandeman, D. C. (1967) Excitation and inhibition of the reflex eye withdrawal of the crab Carcinus. J. Exp. Biol. 46: 475-485.

Tully, T., and W. G. Quinn (1985) Classical conditioning and retention in normal and mutant Drosophila melanogaster. J. Comp. Physiol. A $157: 263-277$. 